\title{
Design and synthesis of heterocycle fused enediyne prodrugs activable at will
}

\author{
Luca Banfi, Andrea Basso, Giuseppe Guanti,* and Renata Riva \\ Università degli Studi di Genova, Dipartimento di Chimica e Chimica Industriale \\ Via Dodecaneso 31, 16146 Genova (Italy) \\ E-mail:guanti@chimica.unige.it
}

\begin{abstract}
In this account we report our efforts to prepare simplified analogues of natural occurring enediynes during the last ten years. We have succeeded in preparing two different classes of such compounds were the embedded 10-membered enediyne system is fused either with a $\beta$-lactam ring or with an epoxide. Biological tests on these compounds have demonstrated that some representatives are able to cleave the double strand of DNA even at very low concentrations; efforts have also been devoted to make these compounds active only under controlled conditions.
\end{abstract}

Keywords: Enediynes, antitumor compounds, lactenediynes, Dynemicin analogues

\section{Contents}

Introduction

1. $\beta$-Lactam-fused enediynes: Lactenediynes

2. Symplified Dynemicin analogues

Conclusions

\section{Introduction}

The enediyne antitumor antibiotics ${ }^{1,2}$ are a small family of natural compounds, whose structure has been disclosed in the $80 \mathrm{~s} .{ }^{3,4}$ The novel molecular architecture of these compounds was intriguing, since conjugated enediynes were considered to be rather unstable structures, and they were not expected to be found in nature. Moreover, natural enediynes were found to be highly cytotoxic and characterized by a potent antitumor activity; their unprecedented mode of action opened the door to the development of several artificial enediynes endowed with interesting biological properties. 
In all natural enediynes the conjugated system is embedded usually in a 10 -membered ring, and in some cases in a 9-membered ring.

Calicheamicin $\gamma_{1}^{\prime}$ (1) from Micromonospora echinospora spp. calichensis is one of the main representatives of the family of enediynes (Figure 1). ${ }^{4,5}$ Apart from the enediyne moiety, this molecule contains several unusual structural features, such as the acyl enamine and the trisulfide in the aglycon, the hexasubstituted aryl ring, the thioester and the hydroxylamine glycosidic linkages in the saccharide. Another member of this family, Dynemicin A (2) ${ }^{6,7}$ (Figure 1) from Michromonospora chersina, contains a novel combination of an anthraquinone moiety fused with a 10-membered bridging enediyne ring.

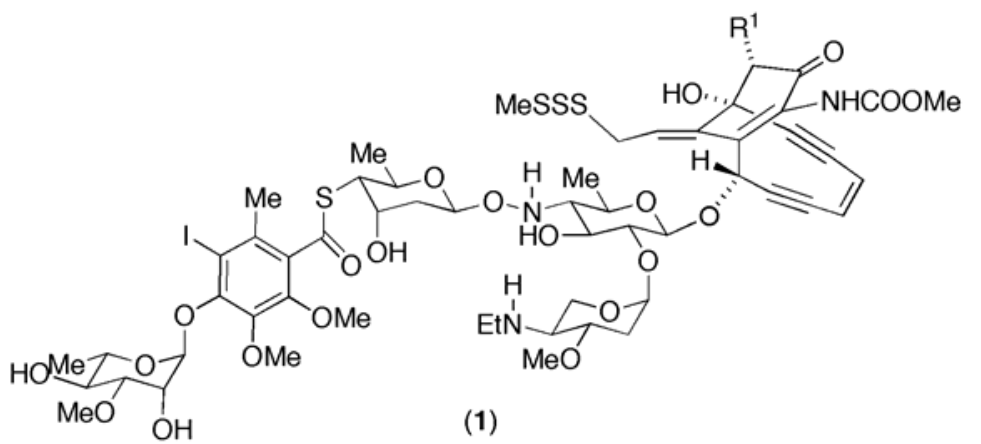

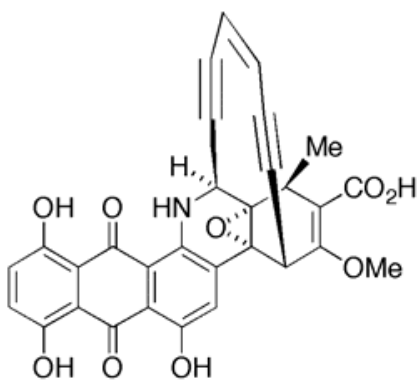

(2)

\section{Figure 1}

These compounds are extremely cytotoxic and cause both in vitro single- and doublestranded DNA cuts of supercoiled plasmids. Obviously the damage caused to the cell by double cut is by far more fatal than that caused by a single cut.

At the basis of the activity of natural enediyne molecules is the key transformation of the 3-ene-1,5-diyne system (3) into a diradical benzenoid species (4) (a p-benzyne). ${ }^{8}$ This thermal rearrangement was disclosed in the early '70s by Masamune ${ }^{9}$ and Bergman, ${ }^{10}$ and it is commonly called the Bergman cycloaromatization (Scheme 1).

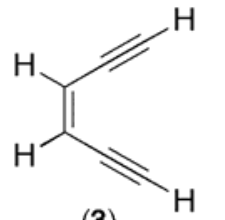

(3)

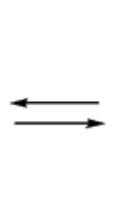

-

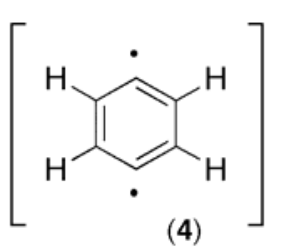

(4)

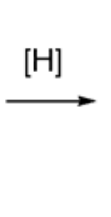

Scheme 1. The Bergman cycloaromatization

Calicheamicin is a masterpiece of nature's ingenuity. It has been compared to a smart bomb equipped with: a) a delivery system which is responsible for a strong and specific complexation with DNA. This system is represented by the oligosaccharide unit; b) a warhead (the enediyne moiety) that, transformed into the highly reactive diradical species is able to attack 
the DNA strands. The bifunctional nature of the diradical allows not only a single strand cleavage, but even lethal double cleavage if the geometry of the complex DNA-enediyne allows that; ${ }^{11}$ c) a safety catch that prevents the enediyne Bergman reaction, by imposing a structural restraint to its cycloaromatization. In this way the warhead does not explode until a particular chemical event takes place. In Calicheamicin the safety catch is represented by the enamine double bond; d) a chemical trigger, that mediates the removal of the safety catch and therefore unleashes the high reactivity of the enediyne. In (1) the trigger is the trisulfide group.

Acyclic Z 3-ene-1,5-diynes such as (3), undergo the classical Bergman thermal cycloaromatization only at elevated temperatures (above $200{ }^{\circ} \mathrm{C}$ ). However, Nicolaou has shown that, by including the enediyne in a ring, the steric strain in the substrate may lower the activation energy to such a point that cycloaromatization takes place even at room temperature. Not surprisingly, the crucial ring size was found to be 10 as in the case of most natural enediynes: compound (6) undergoes cycloaromatization at $37^{\circ} \mathrm{C}$ with a $\mathrm{t}_{1 / 2}$ of $18 \mathrm{~h}$, while larger rings were completely stable (Scheme 2). ${ }^{12}$ From this evidence enediynes such as Calicheamicin or Dynemicin, where the unsaturated moiety is included in a 10 -membered ring, should be expected to be poorly stable at room temperature. However in such compounds a structural bias acting as a safety catch is present, thus only after a suitable triggering event the cycloaromatization is allowed to take place.

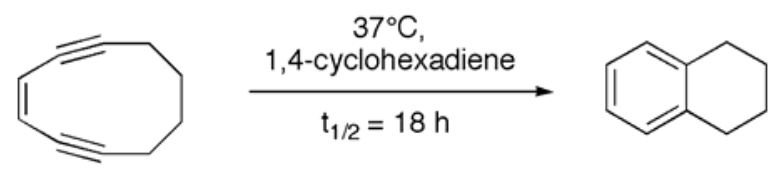

(6)

(7)

Scheme 2. The cycloaromatization of cyclodeca-3-ene-1,5-diyne

Also Dynemicin displays a delivery unit (the anthraquinone), a safety catch (the epoxide) and a trigger (the quinone). Bioreduction of the quinone unleashes two lone-pairs (one on the nitrogen and one on the upper hydroquinone oxygen) which bring about intramolecular epoxide opening. ${ }^{1}$

Although conjugation of the natural enediynes with monoclonal antibodies has led to important clinical results, ${ }^{13}$ the quest for more selective analogues is still an important issue. However, the structural complexity of the natural substances makes de novo total synthesis - as well as semisynthesis - of analogues rather difficult. On the other hand, the knowledge of the chemical foundations of their unique mode of action (based on the triad warhead, safety catch, trigger) enables in principle the rational design of simpler artificial analogues based on the same general concept. ${ }^{14}$ These simpler analogues may be then coupled with several DNA-complexing agents (the delivery unit) in order to assemble enediyne prodrugs endowed with the desired potency and selectivity. 
Taking inspiration from the natural enediynes and taking advantage of molecular modelling calculations, about ten years ago ${ }^{15}$ we have postulated that simple enediynes systems embedded in a 10-membered ring and trans fused with a cyclobutane- or cyclopropane-like moiety would be stable towards cycloaromatization, the cyclic moiety constituting the safety catch. We reasoned that, after opening of the small cycle, cycloaromatization could occurr in a way similar to that of compound (6). The subsequent step was therefore to find suitable cyclobutane and cyclopropane surrogates that could be chemically fused to the enediyne system and be selectively opened under controlled conditions. Our choice fell onto the $\beta$-lactam ring as cyclobutane surrogate and the epoxide ring as cyclopropane surrogate, leading respectively to compound (8) and compound (12) (Scheme 3).

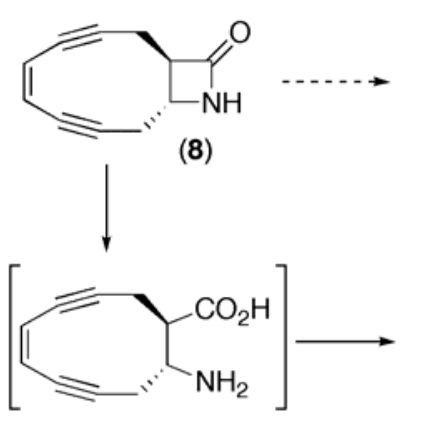

(10)

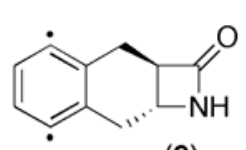

(9)

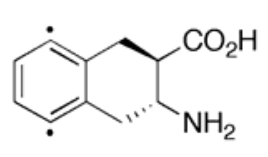

(11)

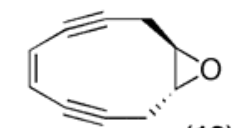

(12)

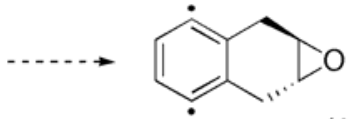

(13)

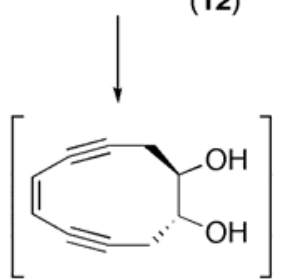

(14)

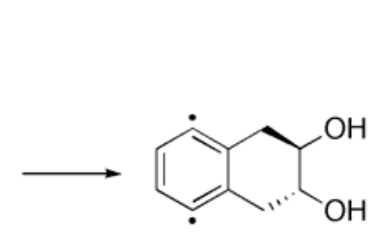

(15)

Scheme 3. 10-Membered cyclic enediynes fused with a $\beta$-lactam and an epoxide ring

We will now illustrate separately these two approaches, starting from the family of $\beta$ lactam fused enediynes that were elaborated first in our laboratories and were named lactenediynes. Since we had no information on the influence of absolute configuration on biological activity, all these compounds were synthesized in racemic form.

\section{1. $\beta$-Lactam-fused enediynes: Lactenediynes}

The simplest member of this family is compound (8), ${ }^{16}$ where only the warhead and the safety catch are displayed; compound (8) was assembled, as all the other members of this family, starting from the $\beta$-lactam ring: it was clear in fact that the safety catch ring had to be assembled first in order to prevent cycloaromatization of the enediyne. In principle it is possible to assemble the enediyne moiety and the 10-membered ring at the same time or as two separate events: in this case the first strategy seemed more promising. Starting from commercially available 4acetoxyazetidinone (16), double propargylation gave the desired trans derivative (18) via intermediate monopropargylated (17). The $p$-methoxybenzyl groups were then removed and the resulting bis propargylic alcohol derivative was oxidized under Swern conditions to give (19). Compound (20) was obtained with a stereoselective reductive pinacol coupling catalized by a 
vanadium complex, followed by thionocarbonate formation and reduction with an activated phosphine to give lactenediyne (21), analogous of (8) apart from the silyl protecting group on the nitrogen atom, in an overall 13\% yield (Scheme 4 ).

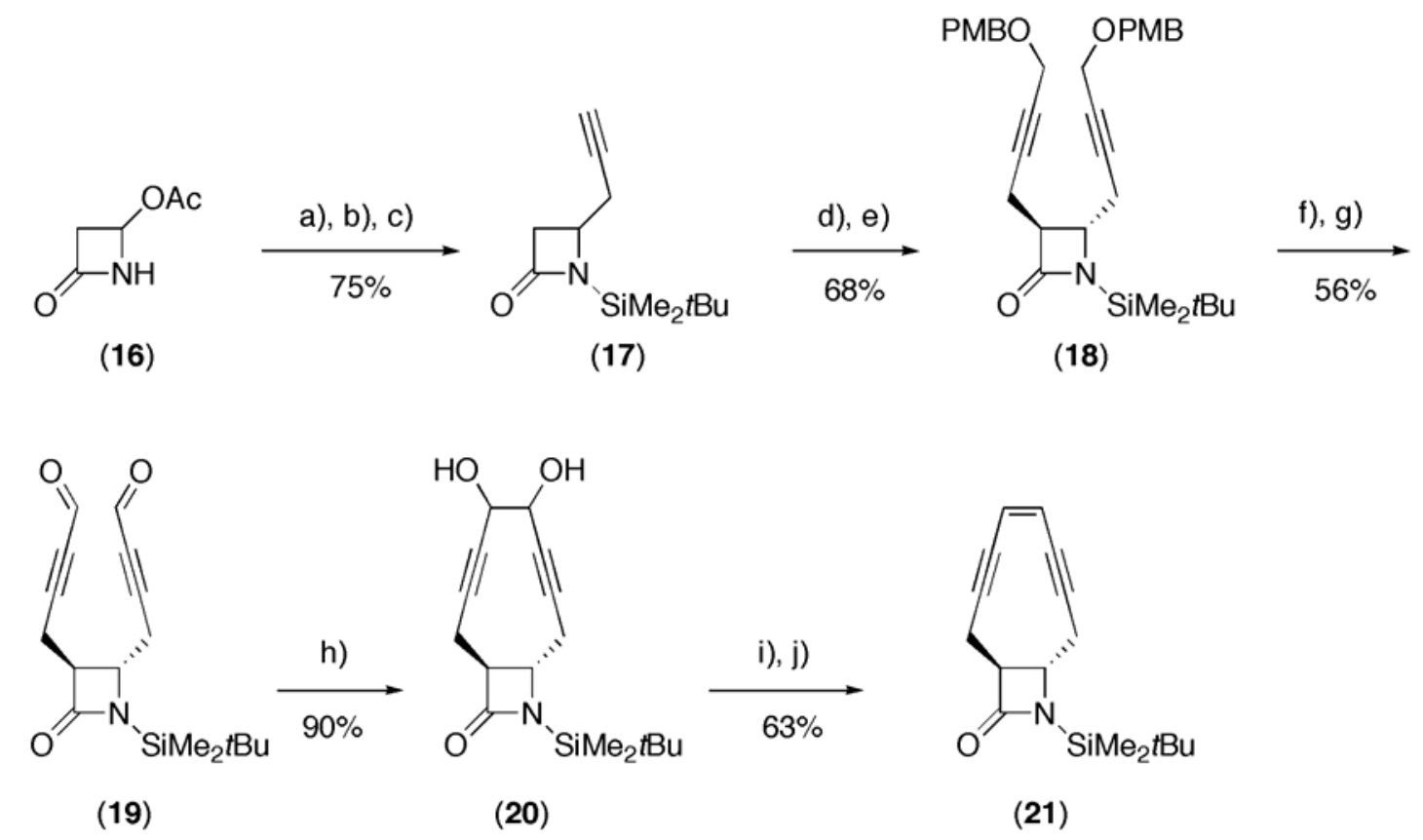

Scheme 4. Reagents: a) $\mathrm{PhSO}_{2} \mathrm{Na}$; b) propargyl magnesium bromide; c) $\mathrm{Me}_{2} t \mathrm{BuSiCl}$; d) LDA, $p$ methoxybenzyloxymethylchloride; e) LDA, 4-bromo-1-(p-methoxybenzyl)oxy-2-butyne; f) DDQ; g) $\left.(\mathrm{COCl})_{2}, \mathrm{EtN}(\mathrm{iPr})_{2}, \mathrm{DMSO} ; \mathrm{h}\right)\left[\mathrm{V}_{2} \mathrm{Cl}_{3}(\mathrm{THF})_{6}\right]_{2} \mathrm{Zn}_{2} \mathrm{Cl}_{6} ;$ i) thiocarbonyl diimidazole; j) $P$-phenyl- $N, N$-dimethyl-1,3-diaza-2-phospholidine.

Although this synthesis was relatively short and straightforward (it is always to be taken into consideration that the synthesis of polyunsaturated medium size rings is often not an easy task), the molecule obtained displayed only two of the four essential features of natural enediynes: the warhead and the safety catch. The $\beta$-lactam, being unactivated is too stable to undergo ring opening under physiological conditions. Therefore an activating substituent or a triggering device must be added. Moreover it is also important, in order to optimize activity, to add a delivery unit. Compound (21) is provided with only one attachment point ("handle"), that is the nitrogen atom. It was therefore devised that the introduction of some additional handles on the basic structure would have been highly desirable and an alternative synthetic strategy was designed. ${ }^{17}$ This time the assembly of the conjugated enediyne system was performed first on an acyclic precursor, followed by cyclization to give the 10 -membered ring. This is probably the approach most widely followed in enediyne chemistry and, usually, it takes advantage of the intramolecular addition of an acetylide anion onto an aldehyde. Thus, in order to use this approach we had to prepare an acyclic enediyne with an additional primary alcohol that could be oxidized to an aldehyde group in order to effect cyclization. The synthesis started in this case 
from racemic aspartic acid, that, following a known procedure, was transformed into the monosubstituted $\beta$-lactam (22). In a first set of experiments a not removable methyl group was introduced onto the $\beta$-lactam nitrogen: although the nitrogen in this way could not be exploited as handle, it allowed us to explore the subsequent steps of the synthesis on a stable substrate. A stereoselective introduction of the propargyl group, through propargylation of the $\beta$-lactam enolate, was followed. At this point the silyl group at the alkyne of (23) could be removed selectively by the action of silver nitrate, without affecting the silyl group at oxygen, giving (24). The acyclic enediyne (25) was assembled via the stereospecific Sonogashira reaction with 1chloro-4-(trimethylsilyl)but-1-en-3-yne. The final cyclization was carried on with the Nozaki reaction, so we converted in two high yielding steps the silyl enediyne into iodo enediyne (26). After oxidation of the alcohol, treatment with chromous chloride gave the desired products (27) in very good yields (Scheme 5). A similar route was followed also to prepare a second lactenediyne bearing at the $\beta$-lactam nitrogen a silyl removable group, in order to render such nitrogen atom able to be further elaborated.

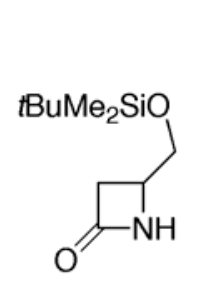

(22)

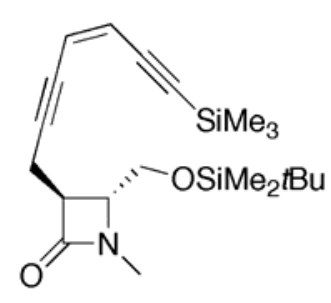

(25)

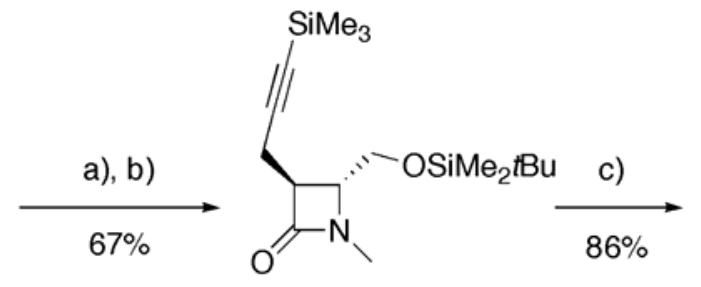

(23)

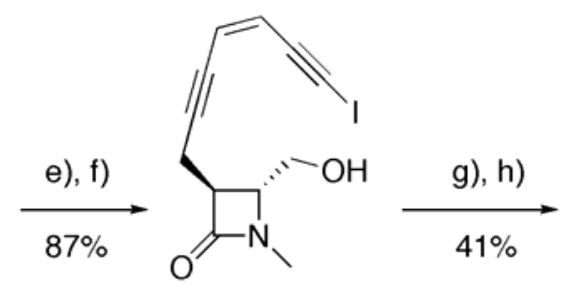

(26)

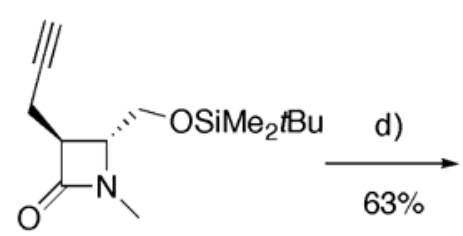

(24)

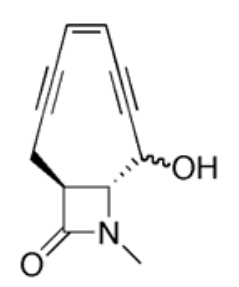

(27)

Scheme 5. Reagents: a) methyl iodide, $\mathrm{KOH}, n \mathrm{Bu}_{4} \mathrm{NBr}$; b) LDA, trimethylsilylpropargyl bromide; c) silver nitrate, $\mathrm{KCN}$; d) 1-chloro-4-(trimethylsilyl)but-1-en-3-yne, $\mathrm{Pd}(\mathrm{PhCN})_{2} \mathrm{Cl}_{2}$, $\mathrm{CuI}$, piperidine; e) silver nitrate, N-iodosuccinimmide; f) $\mathrm{HF}$, water; g) $(\mathrm{COCl})_{2}, \mathrm{EtN}(i \operatorname{Pr})_{2}$, DMSO; h) $\mathrm{CrCl}_{2}, \mathrm{NiCl}_{2}$.

The overall yield of this synthesis, although longer than the previous one, was good, and it allowed us to prepare the final enediyne (27), as well as its silylated analogue, in a gram scale.

Also in the case of lactenediynes with two additional handles a similar approach for the synthesis of the cyclic enediyne system was followed, ${ }^{18}$ however this time the $\beta$-lactam (29) was assembled more convergently, by the use of a Staudinger condensation with (28), having a styryl 
group as synthetic equivalent of a hydroxymethyl group. The choice of the protecting groups at the tertiary alcohol and at the nitrogen was crucial. Actually, after propargylation to give (30), $p$ methoxybenzyl groups had to be removed and changed into silyl protecting groups to give (31), since their removal was found to be troublesome at later stages of the synthesis. A key step of this synthesis was the chemoselective ozonolysis of the double bond of (31) in the presence of the triple one. This nearly unprecedented reaction was found to work well, provided that the terminal alkyne was unprotected. From compound (32) the synthesis was carried out according to the previous one, via key intermediate (33), to give the desired lactenediyne (34) equipped with three handles (Scheme 6).

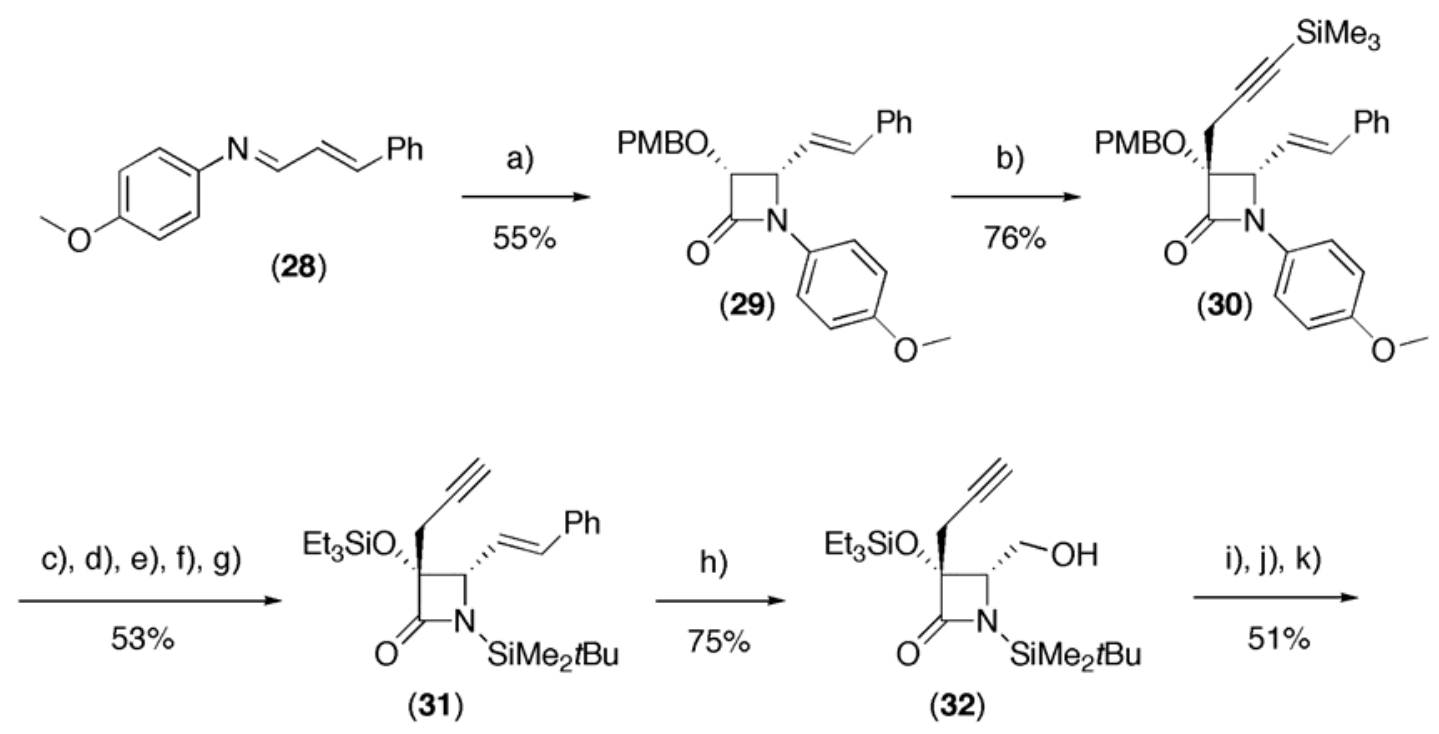

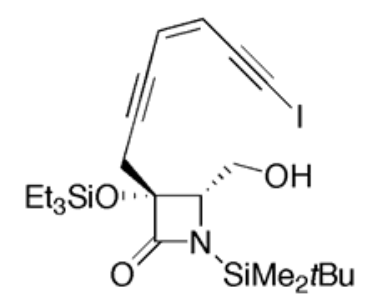

(33)

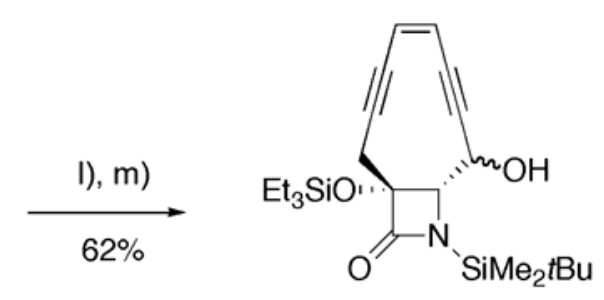

(34)

Scheme 6. Reagents: a) pMeO-benzyloxyacetic acid, $\mathrm{PhOPOCl}_{2}, \mathrm{Et}_{3} \mathrm{~N}$; b) LDA, trimethylsilylpropargyl bromide; c) DDQ; d) Et ${ }_{3} \mathrm{SiOTf}, 2$,6-lutidine; e) $\left(\mathrm{NH}_{4}\right)_{2} \mathrm{Ce}\left(\mathrm{NO}_{3}\right)_{6}$; f) $\mathrm{Me}_{2}$ BuSiOTf, 2,6-lutidine; g) silver nitrate, $\mathrm{KCN}$; h) $\mathrm{O}_{3}, \mathrm{NaBH}_{4}$; i) 1-chloro-4(trimethylsilyl)but-1-en-3-yne, $\mathrm{Pd}(\mathrm{PhCN})_{2} \mathrm{Cl}_{2}, \mathrm{CuI}$, piperidine; $\mathrm{j}$ ) silver nitrate, $\mathrm{KCN}$; $\mathrm{k}$ ) iodine, morpholine; 1) $\left.(\mathrm{COCl})_{2}, \mathrm{EtN}(\mathrm{iPr})_{2}, \mathrm{DMSO} ; \mathrm{m}\right) \mathrm{CrCl}_{2}, \mathrm{NiCl}_{2}$.

When we moved to test the biological activity of these compounds we found that they were stable in the dry state (quite unusual for enediyne molecules) and did not give any 
cycloaromatization products even if heated at $100^{\circ} \mathrm{C}$. A complete different behaviour was found when the $\beta$-lactam ring was opened; in fact cycloaromatization could occur even under physiological conditions. However, to our surprise, the $\beta$-lactam ring was found more stable than expected and relatively strong basic conditions were required in order to open it when the nitrogen was free or alkylated. The scarce reactivity could be overcome binding an activating group to the nitrogen: in this case $\beta$-lactam opening and subsequent cycloaromatization could be achieved at $37^{\circ} \mathrm{C}$ even at physiological $\mathrm{pH} .{ }^{19}$ These activated lactenediynes were therefore tested against plasmid DNA, giving encouraging results, since, even in the absence of any delivery unit able to complex the DNA strands, these compounds were able to provoke single and double strand break in plasmid DNA (the ratio between single and double break was about 15:1) with an overall activity about ten times higher than that of simple monocyclic enediynes.

The activity of these three types of lactenenediynes could be improved by taking advantage of the "handles" for appending DNA complexing substructures. Such complexing substructures could be attached either at the nitrogen, together with the activating substituent, or at one of the hydroxy groups, and about 25 conjugated derivatives have been prepared so far and tested in vitro against plasmid DNA.

Several of these conjugated derivatives proved to be more active than the compounds devoid of a delivery unit. One of them was found to be about 3-5 times more potent in overall break, but with a higher percentage of double break. So the potency relative to double break, that is the most lethal one, was approximately 15 times higher. Double break was still evident at 1 micromolar concentration. This compound is probably the most potent among artificial monocyclic or ortho-fused enediynes prepared so far.

The activating substituents placed on nitrogen in these compounds are not selective. They are expected to activate the $\beta$-lactam towards solvolysis in any kind of cell. The lactenediynes of this type are therefore not selective against cancer cells, although a certain degree of selectivity may be realized by the appropriate choice of the DNA-complexing substructures. We have therefore decided to embark ourselves in a project aimed at finding a way to activate the $\beta$ lactam towards opening in a selective way; our idea was to place a side arm with an amino group able to open the $\beta$-lactam ring via an intramolecular transamidation, forming a seven membered lactam no longer able to stabilize the enediyne. The amino group can be conveniently protected with various enzymatically removable groups and set free only in the presence of a tumor cell. These strategies have already been applied in other fields, and they are generally called prodrug enzyme therapies, since an enzyme (that can be already present in large amount in the tumor cell or it can be vehiculated with an antibody or it can be produced in the tumor cell via introduction of the opportune gene) is responsible for the transformation of a prodrug into a drug. At the outset of this work very little was known on the intramolecular nucleophilic opening of simple monocyclic $\beta$-lactams; therefore a thorough study on model $\beta$-lactam compounds devoid of the enediyne moiety was firstly carried on. ${ }^{[20]}$ It was found that a side chain bonded to the nitrogen was more efficient that a side chain bonded at C-3 of the $\beta$-lactam. It was also observed that 
accelerating effects were given by a nearly neutral buffer and an alkoxy group at position 3 (Scheme 7).

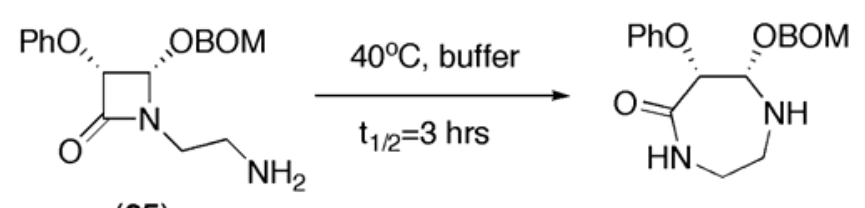

(35)

(36)

\section{Scheme 7}

In a parallel way, it was also checked that the seven membered lactam was indeed no longer able to stabilize the enediyne, and indeed when model compound (37) was heated in THF at reflux in the presence of a hydrogen donor, compound (39) was isolated (Scheme 8).

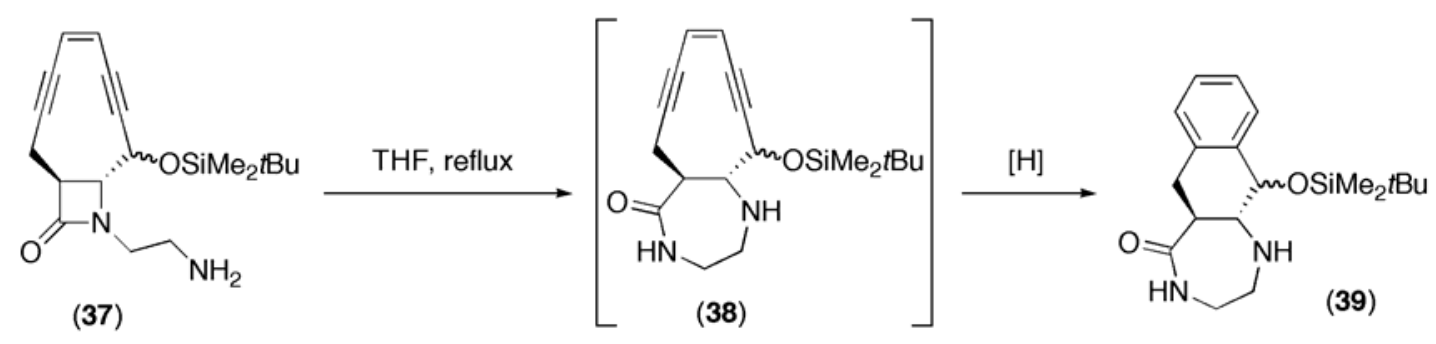

\section{Scheme 8}

However, when we moved to explore the possibility of performing the transamidation process under physiological conditions we found that lactenediynes such as (37) were not sufficiently reactive. So we devised and realized a de novo synthesis of lactenediynes bearing a methoxy group at C-1 and a tethered protected hydroxyl at nitrogen. ${ }^{21}$ Also this synthesis starts with a Staudinger condensation and involves, as key step, a chemoselective ozonolysis of a double bond in the presence of a triple one. It allowed multigram preparation of the final lactenediyne. When the side-chain hydroxyl group was substituted with an amino group, the resulting compound (41) (Scheme 9), showed some activity, although it resulted 10 times less potent that unselectively activated compounds and it was not able to produce double cut of the DNA strands. Anyhow, this compound permitted to demostrate that it is possible to tune the activity of the lactenediynes by the presence or absence of a protection on the amino group (in fact compound (40) where the amino group was protected, was not active at all against plasmid DNA). Although the low potency of (41) makes it not very useful, it could be possibly improved by addition of suitable DNA complexing agents. However, experiments have shown that the main reason for low reactivity of (41) is the very slow intramolecular opening of the $\beta$-lactam ring. 


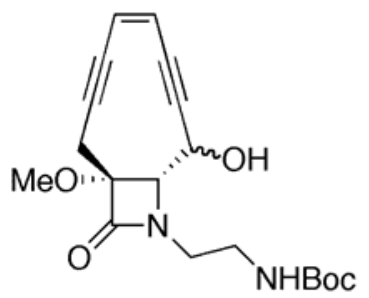

(40)

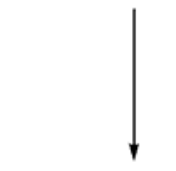

no DNA cleavage

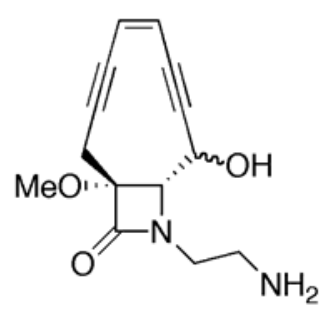

(41)

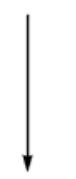

single strand DNA break

\section{Scheme 9}

Also for this reason, our present work is aimed at the synthesis of new types of lactenediynes, such as those reported in Figure 2. From molecular modelling calculations these new lactenediynes are expected to be more tensioned and therefore they should be more reactive, rendering the selective opening of the $\beta$-lactam more easily achievable.

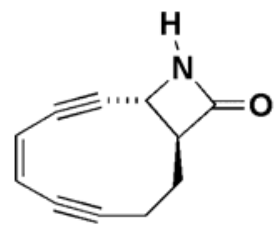

(42)

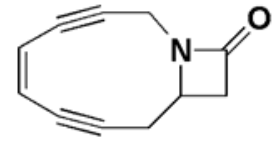

(43)

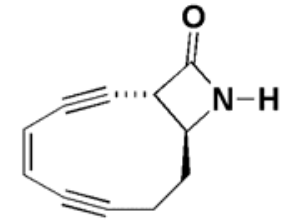

(44)

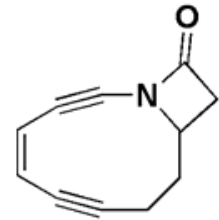

(45)

\section{Figure 2}

We have successfully prepared members of the general family represented by (43). ${ }^{22}$ They however were found to be not very stable at the dry state and this fact may prevent their pharmaceutical applications. More recently we have carried out two alternative approaches to structures (42). ${ }^{23}$ In both cases, however, the final cyclization step failed to provide the desired monomer. In one case we could isolate various stereoisomeric cyclodimers. Thus the steric strain in this case probably prevents successful cyclization. These cyclodimers may anyhow find applications as host-guest systems.

\section{Symplified Dynemicin analogues}

In parallel with the research project on lactenediynes we have also investigated the possibility of preparing 10-membered cyclic enediynes fused with an epoxide ring, in order to prepare 
simplified analogues of the natural compound Dynemicin. Similar compounds had already been reported by Isobe, ${ }^{24}$ in which the quinone moiety, the phenolic hydroxyl group and the cyclohexene ring were lacking. A triggering mechanism alternative to the reduction of the quinone moiety had to be introduced, and this was identified with the introduction of a protecting group on the nitrogen atom: in the absence of the quinone, in fact, the nucleophilic nitrogen can assist the solvolysis of the epoxide and therefore the cycloaromatization can occur. However, if the nitrogen is protected as a carbamate, its nucleophilicity is very much reduced and the compound is stable; deprotection of the carbamate can be therefore exploited as the triggering event. Moreover, such compounds showed a reduced activity, which can be most likely attributed to the presence of a quaternary carbon bearing a hydroxy group at the propargylic position $\alpha$ to the benzylic terminus of the epoxide, as a consequence of the strategy employed for the generation of the cyclic enediyne (by cyclization of the enediynic acetylide onto a ketone). We therefore planned a different diastereoselective strategy, ${ }^{25}$ outlined in Scheme 10, that started with a regio- and diastereoselective nucleophilic addition of magnesium acetylides onto 2-(4quinolyl)propan-1-ol (46). ${ }^{26}$ After deprotection of the alcoholic function the two epimers $(\mathbf{4 7 a}, \mathbf{b})$ were separed by chromatography and the subsequent transformations were carried on the two isomeric compounds separately (only one epimer is shown in the Scheme). The introduction of the second alkyne moiety was performed using the Corey-Fuchs procedure on the corresponding aldehyde. The resulting vicinal dibromide (48a) was transformed into the terminal alkyne (49a) by treatment with $n$-BuLi. The subsequent steps served to introduce the epoxide ring and to give the bis(iodide) derivative (50a), that was cyclized to the enediyne system (51a) via a Stille double coupling. This reaction was deeply influenced by the position of the methyl group with respect to the other stereocenters and only (51a) was obtained in excellent yield (its epimer was obtained in $60 \%$ yield).

In order to be able to remove the carbamate under physiological conditions, we transformed the stable phenyl carbamate into the much more labile 2-phenylsulfonylethyl carbamate. $^{27}$ The nucleophilic substitution promoted by phenylthioethylate, followed by oxidation to sulfonyl group gave (52a), that was demonstrated to give the desired cycloaromatisation at physiological $\mathrm{pH}$, thanks to the facile $\beta$-elimination process, followed by removal of the carbamate moiety. Unexpectedly the same sequence could not be reproduced on the epimer of (51a). The biological activity of (52a) against DNA was extremely interesting; in fact it is probably the most active Dynemicin artificial analogue and has the highest double to single cleavage ratio of all artificial enediynes prepared so far. 
<smiles>CSC#C[C@H]1C=C([C@H](C)CO)c2ccccc2N1C(=O)Oc1ccccc1</smiles><smiles>CC#C[C@H]1C=C(C(C)C=C(Br)Br)c2ccccc2N1C(=O)Oc1ccccc1</smiles>

(48a)<smiles></smiles>

(50a)<smiles>C#CC(C)C1=C[C@H](C#CC)N(C(=O)O)c2ccccc21</smiles>

(49a)<smiles>CC(C)C#C/C=C\C#CC1CN(C(=O)Oc2ccccc2)c2ccccc21</smiles>

(51a)

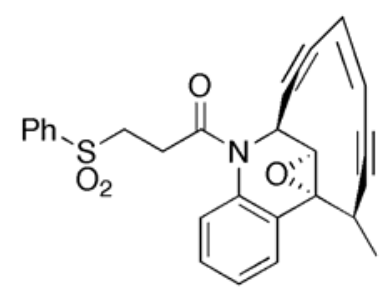

(52a)

Scheme 10. Reagents: a) trimethylsilyl magnesium bromide acetilide, $\mathrm{ClCO}_{2} \mathrm{Ph}$; b) $\mathrm{HF}$; c) $(\mathrm{COCl})_{2}, \mathrm{EtN}(i \mathrm{Pr})_{2}, \mathrm{DMSO}$; d) $\mathrm{CBr}_{4}, \mathrm{PPh}_{3}$; e) $n \mathrm{BuLi}$; f) $\mathrm{NaHCO}_{3}$; g) $m \mathrm{CPBA}$; h) $N-$ iodosuccinimide, $\mathrm{AgNO}_{3}$; i) (Z) $\mathrm{Me}_{3} \mathrm{SnCH}=\mathrm{CHSnMe}_{3}, \mathrm{Pd}\left(\mathrm{PPh}_{3}\right)_{4}, \mathrm{LiCl}$; j) $\mathrm{PhS}\left(\mathrm{CH}_{2}\right)_{2} \mathrm{OH}, \mathrm{NaH}$; k) $m \mathrm{CPBA}$.

Although such compound displayed very promising biological activities, removal of the quinone moiety deprived this simplified analogue of a part able to complex the DNA strands. We therefore decided to elaborate a strategy alternative to the one outlined above, in which the introduction of some handles, as in the case of the lactenediynes, could allow for the bounding of additional parts like delivery units or complexing agents. Moreover, the strategy that was 
elaborated in our laboratories was also the first example of stereoselective synthesis of such compounds. $^{28}$

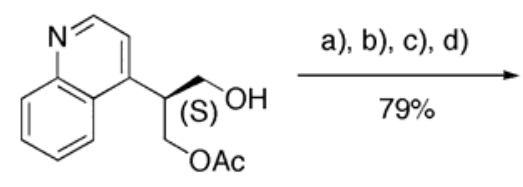

(53)

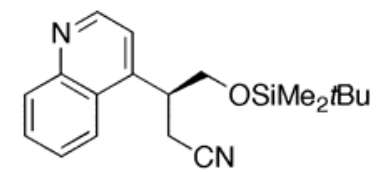

(54)
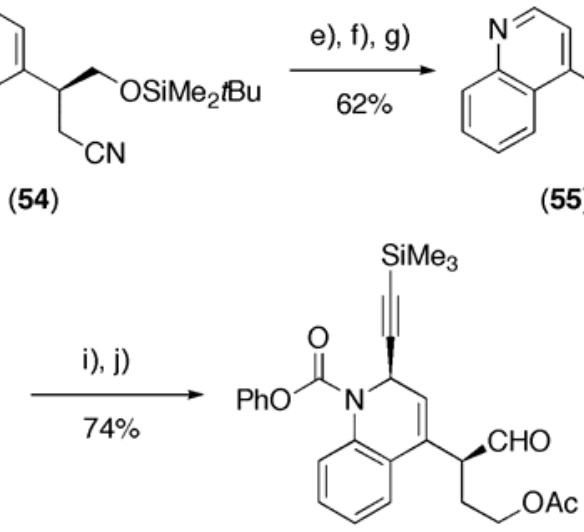

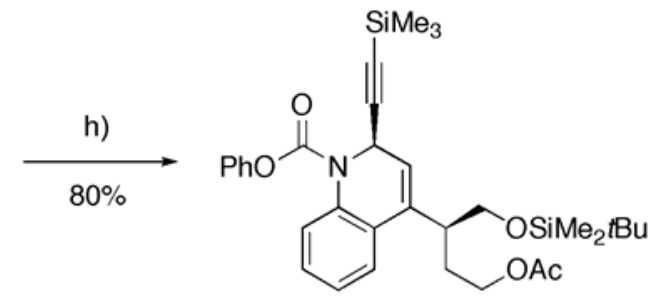

(56)

(57)

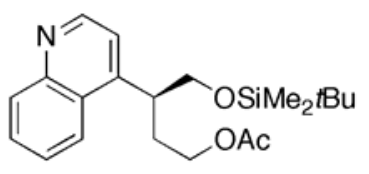

(55)

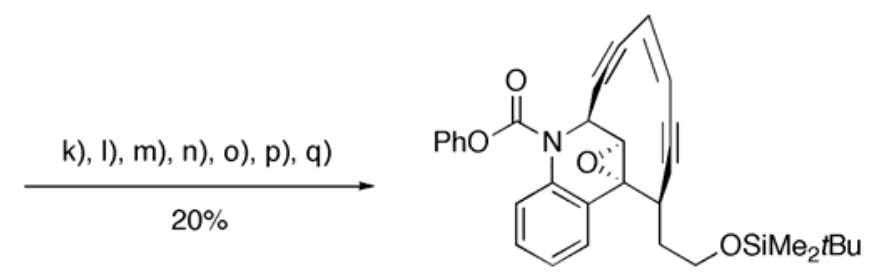

(58)

Scheme 11. Reagents: a) $\mathrm{Me}_{2} t \mathrm{BuSiCl}$, imidazole; b) $\mathrm{KOH}$; c) $\mathrm{TsCl}$, pyridine; d) $\mathrm{KCN}, \mathrm{BBu}_{4} \mathrm{NI}$; e) DIBALH; f) $\mathrm{NaBH}_{4}$; g) $\mathrm{Ac}_{2} \mathrm{O}$, pyridine; h) trimethylsilyl magnesium bromide acetylide, $\mathrm{ClCO}_{2} \mathrm{Ph}$; i) $\mathrm{HF}$; j) $(\mathrm{COCl})_{2}, \mathrm{EtN}(i \mathrm{Pr})_{2}, \mathrm{DMSO}$; k) $\mathrm{CBr}_{4}, \mathrm{PPh}_{3}$; l) nBuLi; m) $\mathrm{K}_{2} \mathrm{CO}_{3}$; n) $\mathrm{Me}_{2} \mathrm{BuSiCl}$, imidazole; o) mCPBA; p) iodine, morpholine; q) (Z) $\mathrm{Me}_{3} \mathrm{SnCH}=\mathrm{CHSnMe}_{3}$, $\mathrm{Pd}\left(\mathrm{PPh}_{3}\right)_{4}, \mathrm{LiCl}$.

Our approach started from monoacetate (53), ${ }^{29}$ easily accessible on large scale by an efficient chemoenzymatic methodology. Although protecting group elaboration could allow us to work in principle on both the synthetically equivalent side arms, it was chosen to carry on the reaction sequence on the $(\mathrm{S})$ enantiomer shown in the Scheme, that possesses the same configuration of natural Dynemicin. Elongation of one of the two arms was obtained via nitrile intermediate (54), that ensured a one-carbon homologation, fundamental for the subsequent transformations to work. Trimethylsilyl magnesium acetylide addition to the quinoline ring of (55) in the presence of phenyl chloroformate, following our previously reported protocol, proceeded in excellent yield and unusually high stereoselectivity to give (56) in $80 \%$ yield, together with $15 \%$ of the other diastereoisomer. The hydroxymethyl group was then converted into an aldehyde and the subsequent steps were performed on (57) according to the conditions previously determined for the preparation of (52a), giving the desired Dynemicin analogue (58) in a $5.5 \%$ overall yield over 17 synthetic steps (Scheme 11). 
Although this compound has not been biologically tested yet, neither has the handle being exploited to bound any complexing agent, in analogy with (52a), we are expecting to find promising results in the near future. Moreover, with our stereoselective synthesis, we will be able to evaluate the effects of absolute configuration on the biological activity.

\section{Conclusions}

During the last ten years our efforts have been devoted to study the chemical and biological properties of two new classes of rationally designed artificial enediynes. These studies have produced many interesting preliminary results and these results have been elaborated further to improve the activity of such compounds as it has been illustrated in this account; more improvements are expected for the near future, taking advantage of the gained experience. Our efforts will be now directed towards the design of novel lactenediynes and Dynemicin analogues able to be intramolecularly activated under controlled conditions, whose principles have been outlined in the previous pages. We will hopefully end up with an "intelligent" drug able to display its lethal properties only in the presence of the desired target at the desired moment.

\section{Acknowledgements}

The authors are grateful to all the students who participated at the "enediyne project" and whose names are reported in the papers cited throughout the text. Without their enthusiasm and skills this work would not have been realised.

\section{References}

1. K. C. Nicolaou, Angew. Chem., Int. Ed. 1991, 32, 1387.

2. A. L. Smith, K. C. Nicolaou, J. Med. Chem. 1996, 39, 2103.

3. K. Edo, M. Mizugaki, Y. Koide, H. Seto, K. Furihata, N. Otake, N. Ishida, Tetrahedron Lett. 1985, 26, 331 .

4. J. S. Thorson, E. L. Sievers, J. Ahlert, E. Shepard, R. E. Whitwam, K. C. Onwueme, M. Ruppen, Curr. Pharm. Design 2000, 6, 1841.

5. M. D. Lee, G. A. Ellestad, D. B. Borders, Acc. Chem. Res. 1991, 24, 235.

6. N. Oku, S. Matsunaga, N. Fusetani, J. Am. Chem. Soc. 2003, 125, 2044.

7. A. G. Myers, N. J. Tom, M. E. Fraley, S. B. Cohen, D. J. Madar, J. Am. Chem. Soc. 1997, $119,6072$.

8. F. Stahl, D. Moran, P. V. Schleyer, M. Prall, P. R. Schreiner, J. Org. Chem. 2002, 67, 1453. 
9. N. Darby, C. U. Kim, J. A. Salaün, K. W. Shelton, S. Takada, S. Masamune, J. Chem. Soc., Chem. Commun. 1971, 1516.

10. T. P. Lockhart, R. G. Bergman, J. Am. Chem. Soc. 1981, 103, 4091.

11. R. A. Kumar, N. Ikemoto, D. J. Patel, J. Mol. Biol. 1997, 265, 187.

12. K. C. Nicolaou, G. Zuccarello, C. Riemer, V. A. Estevez, W.-M. Dai, J. Am. Chem. Soc. 1992, 114, 7360.

13. P. R. Hamann, L. M. Hinman, I. Hollander, C. F. Beyer, D. Lindh, R. Holcomb, W. Hallett, H.-R. Tsou, J. Upeslacis, D. Shochat, A. Mountain, D. A. Flowers, I. Bernstein, Bioconjugate Chem. 2002, 13, 47.

14. M. E. Maier, Synlett 1995, 13.

15. L. Banfi, G. Guanti, Angew. Chem., Int. Ed. Engl. 1995, 34, 2393.

16. L. Banfi, A. Basso, G. Guanti, Eur. J. Org. Chem. 2000, 939.

17. L. Banfi, A. Basso, G. Guanti, Tetrahedron 1997, 53, 3249.

18. L. Banfi, G. Guanti, Tetrahedron Lett. 2002, 43, 7427.

19. L. Banfi, G. Guanti, in Italian Patent, 2002.

20. L. Banfi, G. Guanti, M. Rasparini, Eur. J. Org. Chem. 2003, 1319.

21. L. Banfi, G. Guanti, Eur. J. Org. Chem. 2002, 3745.

22. L. Banfi, G. Guanti, Eur. J. Org. Chem. 1998, 1543.

23. L. Banfi, A. Basso, G. Guanti, M. Paravidino, R. Riva, C. Scapolla, submitted 2005.

24. T. Nishikawa, M. Yoshikai, K. Obi, T. Kawai, R. Unno, T. Jomori, M. Isobe, Tetrahedron 1995, 51, 9339.

25. G. Guanti, R. Riva, Org. Biomol. Chem. 2003, 1, 3967.

26. G. Guanti, S. Perrozzi, R. Riva, Tetrahedron: Asymmetry 1998, 9, 3923.

27. K. C. Nicolaou, W.-M. Dai, S. V. Wendeborn, A. L. Smith, Y. Torisawa, P. Maligres, C.-K. Hwang, Angew. Chem., Int. Ed. Engl. 1991, 30, 1032.

28. L. Banfi, A. Basso, V. Gandolfo, G. Guanti, R. Riva, Tetrahedron Lett. 2004, 45, 4221.

29. L. Banfi, G. Guanti, A. Mugnoli, R. Riva, Tetrahedron: Asymmetry 1998, 9, 2481. 